\title{
Challenges in accessing medication assisted treatment among opiate users attending a methadone clinic in dar es salaam, tanzania: a qualitative assessment
}

\begin{abstract}
Tanzania was estimated to have 200,000 people who inject drugs and $42 \%$ of them are HIV positive. In response to this, Medication Assisted Treatment (MAT) was started in Tanzania in February 2011 as a pilot program aiming at preventing HIV transmission in this population. Studies in countries that have had this program in place for a long time have shown that MAT users encounter challenges when accessing this treatment which hinder the effort of HIV prevention. However nothing is known about the challenges facing MAT users in Tanzanian context given the fact that the program is the first of its kind in Sub-Saharan African region. The study therefore explored the challenges of accessing MAT services in Tanzania.
\end{abstract}

The study was descriptive cross sectional using qualitative approach and involved indepth interviews among 20 purposefully selected opiate users ( $60 \%$ males and $40 \%$ females) attending Muhimbili Clinic in April 2013 to ascertain challenges against accessing MAT. Opiate users included those who were currently receiving treatment and those who had never used MAT. Data analysis was done using qualitative content analysis which involved development of themes.

Strict admission criterion is the biggest challenge reported because the clinic admits clients who inject opiates only. This made some participants to secretly shift their mode of taking heroin from smoking to injecting for the sake of being accepted by the program. The incentive behind this behaviour was reported being the need to quit drugs. Logistic barriers related mainly to transportation and unemployment were also reported to deter access to treatment. Another important challenge reported to prevent access to treatment was misperception about methadone, that it is a poison that kills slowly. Additional themes included; fear of double discrimination and fear of being caught by police Findings suggest urgent review of the program policy to ensure clients are accepted regardless of their route of drug use, to ensure strong community awareness campaigns to address the issue of misperception and discrimination, and also to link the program to other services like employment programs. These strategies will help to increase access to treatment and eventually decelerate the HIV epidemic.
Volume 3 Issue 2 - 2017

\section{Olimpia Laswai, ${ }^{1,2}$ Stephen Nsimba ${ }^{2}$}

'St John's University of Tanzania, Tanzania

${ }^{2}$ University of Dodoma, Tanzania

Correspondence: Olimpia Laswai, SJUT School of Nursing, Dodoma, P.O.BOX 47, Tanzania, Tel +255 7/3-94l-972, Email olympialaswai@yahoo.com

Received: June 06, 2015 | Published: April 03, 2017

Keywords: medication, disease control, symptomatic treatment, risky behaviors

Abbreviations: HIV, human immunodeficiency virus; AIDS, acquired immune deficiency syndrome; CDC, disease control and prevention; YOVARIBE, youth volunteers against risky behaviors; CHRP-TZ, center for human rights promotion-tz; MNH, muhimbili national hospital

\section{Background}

People dependent on opioids have been reported to have poor physical and mental health, increased risk of human immunodeficiency virus, disturbed family relationship, chronic unemployment, and criminal behaviors. ${ }^{1}$ Methadone have been used in USA for treatment of opioid dependence since the mid-1960s, ${ }^{2}$ Since then its use has been expanded to include other countries like Germany in 1987, Netherlands in 1990, China in 2004, Malaysia in 2005, Indonesia in 2003 and Vietnam in 2009..$^{3-8}$ However no medication assisted treatment in Africa up to 2011 where Tanzania became the first and the only country in sub-Saharan Africa to launch this treatment.
Before this time methadone was considered to be the medicine for the first world only, possibly because the link between heroin and HIV in Africa have emerged only recently. ${ }^{9}$ In Dar es salaam there are an estimated 50,000 injecting drug users and about $42 \%$ of them are living with HIV/AIDS. ${ }^{9}$ Prior to methadone pilot program in Tanzania, people with heroin dependence presenting to the clinics for care and treatment had faced a lot of difficulties since there was no formal treatment modalities established. The mainstay treatment modalities by then were symptomatic treatment and counseling approaches to no treatment at all.

In February, 2011 the first methadone clinic in Africa was established in Dar es Salaam city at the Muhimbili National Hospital and in September 2012 another pilot clinic was set at the Mwananyamala Hospital. Also expansion to Temeke hospital is in final stages. Funding for this programme comes from the U.S. President's Emergency Plan for AIDS Relief (PEPFAR) via the Centers for Disease Control and Prevention (CDC) with technical support from Pangaea Global AIDS 
Foundation. The program has collaboration between the Ministry of Health and Social Welfare, Muhimbili University of Health and Allied Sciences, the Tanzanian Drug Control Commission, and CDC which covers community outreach services and supports four nongovernmental organizations (NGOs) in the Kinondoni district of Dar es Salaam. These NGOs include: Youth Volunteers against Risky Behaviors (YOVARIBE), Center for Human Rights Promotion-TZ (CHRP-TZ), Kimara Peers, and Blue Cross Society of Tanzania.

These NGOs identify and recruit candidates for MAT who are committed to stopping their dependence on opioids and who have taken initiative to seek services. ${ }^{10}$ Globally, many studies have indicated that medication assisted treatment programs encounter successes as well as challenges. ${ }^{5,11}$ However, no such studies have been conducted in Sub-Saharan African countries given the recent introduction of medication assisted treatment in the region. Being a new program and in its pilot phase, service users (opiate users) are likely to encounter challenges when trying to access the services. The study therefore explored the challenges of accessing medication assisted treatment in Tanzania

\section{Methodology}

\section{Study design}

The study design was descriptive cross-sectional using qualitative approach. This design was used because the study sought to understand and describe the challenges of accessing MAT services from the perspectives or view points of the local population it involved. ${ }^{12}$

\section{Study area/setting}

The study was conducted at Muhimbili National Hospital in the Ilala Municipal of Dar es Salaam.

Dar es Salaam is the largest city in Tanzania, with an estimated population of $3,500,000 .^{13}$ It is Tanzania's most important city for both business and government, with high concentrations of trade, manufacturing and other services compared to other parts of the country. Dar es salaam is also a key Indian Ocean port and thus a point of entry for drugs. A few studies have been carried out among IDUs in Dar and confirm that heroin use via injection is an increasing problem in the city. ${ }^{9}$ The medication assisted treatment was launched at Muhimbili National Hospital (MNH) in February 2011 and sixteen months later the same program was launched at Mwananyamala hospital. Mwananyamala MAT clinic being a very new site, the study did not involve this site instead the study was conducted at $\mathrm{MNH}$ only.

\section{Muhimbili National Hospital}

The psychiatry treatment unit of the Department of Psychiatry and Mental Health at the MNH provides services within the Medical Services Division of the hospital that include clinical care and rehabilitation of persons with mental disorders including persons with substance use disorders. The department also provides consultation services for the National Mental Health Programme as contributions to an advisory committee; research in relevant mental health concerns in the Tanzanian context and training for nurses, medical doctors, social workers (practicum placements), occupational therapists (practicum placements) and medical counselors (focus on HIV/AIDS counseling and counseling for substance abuse and dependence).

There is small physical structure at the site for running outpatient clinics for persons with heroin use problems (Muhimbili MAT clinic).
The clinic started with four clients and until April 2013 the clinic had 638 clients with 589 males and 49 females. Since establishment of the clinic until April 2013 there have been 25 deaths, 3 voluntary discharge, 11 involuntary discharge and 150 defaulters. The clinic has 15 workers. One receptionist, two assessment nurses, two social workers, one psychologist, five specialists, two pharmacists and two security guards. The clinic has also trainees in psychology and psychiatry and intern nurses and doctors. The clinic opens daily. The dispensing window opens at 6AM during weekdays and closes at midday and other activities continue until 5PM. On weekends the dispensing window opens at $6 \mathrm{AM}$ and closes at 11AM. The clinics strictly receive clients who inject opiates only. Clients in this clinic receive methadone and treatment for physical illnesses free of charge.

\section{Study population and size}

The study involved 20 opiate users attending Muhimbili methadone clinic. Opiate users included clients on MAT program at the time of study and those who have dropped-out of the program. Drop outs included involuntary discharge and defaulters. Involuntary discharged clients were those discharged/expelled from the program due to misbehaviors' such as fighting, abusing alcohol, abusing workers etc. ${ }^{14}$ On the other hand, defaulters were clients who went missing for 30 or more consecutive days ${ }^{14}$ (Table 1$)$.

Table I Socio-demographic characteristics of service users who participated in the study

\begin{tabular}{|c|c|c|}
\hline & Number & Percent (100\%) \\
\hline \multicolumn{3}{|l|}{ Age } \\
\hline $20-29$ & I & 5 \\
\hline $30-39$ & 15 & 75 \\
\hline $40-49$ & 3 & 15 \\
\hline $50-59$ & I & 5 \\
\hline Total & 20 & $100 \%$ \\
\hline \multicolumn{3}{|l|}{ Sex } \\
\hline Male & 13 & 65 \\
\hline Female & 7 & 35 \\
\hline Total & 20 & $100 \%$ \\
\hline \multicolumn{3}{|l|}{ Education } \\
\hline Primary & 12 & 60 \\
\hline Secondary & 6 & 30 \\
\hline College & 2 & 10 \\
\hline Total & 20 & $100 \%$ \\
\hline \multicolumn{3}{|c|}{ Marital status } \\
\hline Single & 8 & 40 \\
\hline Married & I & 5 \\
\hline Divorced & 2 & 10 \\
\hline Cohabiting & 6 & 30 \\
\hline Separated & 3 & 15 \\
\hline Total & 20 & $100 \%$ \\
\hline
\end{tabular}


Table Continued....

\begin{tabular}{lll}
\hline & Number & Percent (100\%) \\
\hline Employment status & & \\
\hline Employed & 7 & 35 \\
Un employed & 13 & 65 \\
Total & $\mathbf{2 0}$ & $100 \%$ \\
\hline Status at the clinic & & \\
\hline Currently on methadone & 10 & 50 \\
Involuntary discharged & 3 & 15 \\
Defaulters & 7 & 35 \\
Total & $\mathbf{2 0}$ & $100 \%$ \\
\hline
\end{tabular}

\section{Selection of study participants}

The 20 opiate users were purposeful selected through recommendation by the head of the clinic and service providers working at the clinic. This was to make sure that the selected participants were well informed about the MAT services. Opiate users who at the time of the study were using MAT services were invited to participate, as they came for their daily methadone treatment. For service users who dropped out of the program their lists and contacts was obtained from the clinic data clerk and then they were called to be informed about the study, and for those who were interested to participate were invited to come at the Muhimbili MAT clinic and eventually recruited in the study.

\section{Data collection}

Data was collected using in-depth -Interviews which was carried out using Kiswahili language. A total of 20 interviews were conducted with the opiate users at Muhimbili MAT clinic. The number of interviews that were conducted was based on saturation as the researcher continued increasing the sample until the interviews revealed no new information. The interviews were audio recorded for quality control and lasted between 30 minutes to one hour per each interview and were conducted within the clinic compound in a quiet room with only the interviewee and interviewer present. Before the interviews the participants read and signed the informed consent that they had agreed voluntarily to participate. The interviews were semistructured and included a set of open ended probes which were used where necessary. The open ended probes asked the participants about their sociodemographic characteristics, the current situation of MAT service provision and the perceived barriers/challenges in accessing MAT services. All participants were reimbursed a total of 5,000Tshs equivalent to 3.07USD for their transport.

\section{Data analysis}

Data was analyzed using qualitative content analysis. Qualitative content analysis was used to create a picture of challenges facing opiate users as they try to access MAT services..$^{15}$ The Swahili recorded interviews were transcribed and then translated by using forward and back translation method. In doing this the Swahili transcripts were first translated into English by the researcher herself who is bilingual. Then the outcome was translated back to Swahili by another person who is also bilingual. Then the transcripts were then compared with the original Swahili transcripts to assure that the integrity of the data was saved in the translation. The final English transcripts were read through several times to obtain an overall understanding and to gain insight into the meaning of the informants. The whole interview transcripts were used as a single unit of analysis, then meaning units were summarized from the interview into condensed meaning units while preserving the core meaning. ${ }^{16}$ Then the condensed meaning units were abstracted and labelled with a code. Then the various codes were compared based on similarities and differences and sorted into categories. Categories were then sorted into themes. ${ }^{16}$

\section{Ethical consideration}

Ethical clearance was sought from UDOM Human Research and Publication Committee. Permission to conduct the study was sought from the director of Muhimbili National Hospital. Informed consent forms were issued to individual study participants. The components of the informed consent including study purpose, procedures, risks and benefits were reviewed together with the participants. All questions raised by the participant were answered accordingly. The participants were informed that participation into the study was entirely voluntary and that one could withdraw from the study anytime without any bad consequence on the service one was receiving. The participants were informed that all information that had been obtained was entirely confidential and that no data collection instrument would bear their names.

\section{Results}

After analysis of the perceived challenges of accessing MAT services by the opiate users, several themes emerged and are discussed below;

\section{Strict admission criteria}

Currently the Muhimbili MAT clinic receives only opiate users who inject. The need to quit drugs was not only to people who inject opiates but also to people who use opiates using other routes. They both had waited for treatment for so long. Because of this being a very important admission criterion some study participants reported to secretly shift to injection for sometimes until they developed needle marks for the sake of seeking acceptance into the program. Strict admission criterion was mentioned to be an important hindrance to accessing MAT. One participant recalled her experience of how she was recruited into the program. She had the following to say:

When I saw my fellows who used to inject are using methadone and have changed I also wished to change but they told me that I would not be accepted into the program because the program is for injectors only, so I had to shift from sniffing to injecting for four months. After I had my marks I went to YOVARIBE and I started training for one month and when I was ready I signed a contract to start treatment so after this I was referred to MNH where I started my treatment. If I hadn't used my brain, I wouldn't have been in this treatment program today (female 40 years, separated, primary education, unemployed on methadone for eight months).

\section{Logistical difficulties}

Until now take home doses of methadone is not allowed at the Muhimbili MAT clinic. MAT requires its users to attend clinics on a daily basis which poses logistical difficulties for many users. Some opiate users reported coming to the clinic by bus while others on foot. Those coming to the clinic by bus reported making it to the clinic sometimes late despite waking up early in the morning due to huge traffic jam in Dar es Salaam. Many opiate users are unemployed, 
so affording bus fair everyday was reported a problem. Transport difficulties and lack of bus fair was cited as an important challenge in using Muhimbili MAT services. They had the following to say:

Challenges are many. First is how to get to the clinic. I have to really struggle to get daily bus fair. I can't walk from Kinondoni to the clinic. There are times I come to the clinic late due to delay to get fair or even miss a dose due to lack of fair and sometimes if I get a bus fair, the traffic jam is too huge. I am not employed I have to beg all the time for bus fair, my close people are tired already (female 25 years, single, unemployed, defaulter).

Some opiate users reported to have gotten jobs after being in treatment for sometime. The Muhimbili clinic has tried to adjust the opening time for the clinic to be 6:00am to accommodate those who are working but still it becomes very difficult for employed opiate users to go out of the city for business trips given the fact that there are no MAT clinics in other cities and towns. Even with the two clinics available in Dar es Salaam which are located in different districts, clients are not allowed to transfer between these clinics whenever the need arises. They reported as follows:

This treatment is too demanding, I have to come here every day. If you get a job that needs you to be travelling like been a bus conductor. You cannot make it here every day. I asked myself I am doing well with methadone but until when should I be coming here? If we were given a take home dose this would have helped or if same clinic would be available all over the country just like the way heroin is scattered would help too. In addition if you are enrolled at MNH you can only receive services here, you can't access the other clinic at Mwananyamala (Male 36 years, single, secondary education, bus conductor, defaulter)

The study participants also reported to spend much time at the clinic waiting to be given methadone. The clinic has only one window which dispenses methadone to all clients. Time spent was reported to vary considerably between ten minutes to three hours depending on the client's cue number. This was reported as a challenge because many of them wanted to just drop by and take their dose and leave immediately so that they can go to continue with their other activities. They reported as follows:

We are more than four hundred here and yet only one window dispenses methadone. We take much time here. We would appreciate if we have more windows so that we leave early for home (female 30 years, cohabiting, primary education, unemployed, involuntary discharge)

\section{Inappropriate perception about methadone}

The study found that there are some misconceptions about methadone that are affecting or might affect the utilization of MAT services by the study participants. The study participants reported that people in the community has been spreading rumors that methadone is "poison" and that it is a strategy for high income countries to kill all the injecting drug users. They stated that the rumors are so strong in such a way that if someone is weak he/she might not think of using this treatment. Below is a quotation from one drug injector:

Some people in the community tell us methadone is poison. They use a lot of strategies to make sure we quit. If you are weak you might drop out and go out to inject again (male 46 years, divorced, primary education, unemployed on methadone for 27 months).
Misconception about methadone also exist among methadone users themselves especially those who defaulted. Some believe that konyagi is an alternative way to quit drugs. Because of this some of them defaulted from the program to try out this alternative which at the end they found not working. One of defaulters reported as follows:

I was tired of coming here every day, my friend told me konyagi helps to cure the withdrawals. So I thought of trying this alternative way to quit my dependency and avoid getting here every day. This is the reason that made me to drop out. After I dropped out I started injecting again, the konyagi which I thought would help did not help, then I thought the last resort is to come and surrender to methadone again (female 35 years, cohabiting, primary education, unemployed, defaulter).

\section{Harassment from police}

The study participants stated that harassment from police is a significant discouragement for treatment maintenance. The study participants reported to constantly be arrested by policemen and accused of theft even if they are not involved in the theft in order to mask true thieves just because they were once known thieves. The study participants reported that policemen used to benefit from their theft in the past but not anymore since they have changed these days and narrated as follows:

"Even though we have changed, the police cannot let as be free or go if we pass by. For example if theft occurs around our neighbourhood. They will come and arrest us because they were used of us as being thieves. Those times when we used to steal we could bribe them so that they can let us free. So in a way they benefited from our theft. Now that we have changed they don't benefit anymore. Now if somebody else steals and that person bribes them, they will let that person free and come and arrest us purposely to mask that person just because we used to be known thieves (male 50 years, married, primary education, unemployed on methadone for two years).

\section{Double stigma}

The study participants described the kind of stigma directed to them as 'double stigma'. This was explained by the fact that most of them are also HIV positive. Being an addict and also positive gave people an opportunity to stigmatize them even more. The study participants reported that, to be recognized as an addict makes someone to be eligible for double stigma, because the society believes if one injects, there is high probability of being HIV positive as well. The fear for stigma makes them reluctant to access treatment. The stigma was explained by the study participants to include even the providers who provide services to these clients. The doctors are labeled as "doctors for the addicts" and because they can socialize, laugh and cope with these clients, the society thinks these providers are just like the addicts. With this persistent stigma the study participants explained that it is strongly demotivating them together with their healthcare providers. They stated as follows:

It is very hard to accept to be stigmatized. If you are seen around the clinic by a friend is already a confirmation of your status. In 2010 I was diagnosed HIV positive and because of this I was highly stigmatized. I didn't want people to know my second status (addict) because it would add into the stigma I was getting. The services in this clinic started in 2011, I knew of the services but was reluctant to joint until in December 2012 when my condition started deteriorating that's when I joined. This was all because of the fear of being 'double 
stigmatized'. (female 20 years, cohabiting, unemployed, involuntarily discharged).

\section{Difficult adjusting to new life}

The study participants reported doing harmful activities in the past to support their drug use and other activities of daily leaving. When they entered in the treatment program, they found themselves changing, they no longer worry for drugs so they can't see the reason for continuing to steal anymore. Many of them are not employed; they now worry about how they can manage their daily lives like to even manage a daily meal. They reported to have difficult adjusting to this change in their lives and they thought that this can predispose them in their old lifestyle again and so dropout from treatment. One of the drug injectors narrated that:

When I was injecting I didn't have a job but I was never running short of money because my mind could tell me to steal all the time. But now that I am on methadone I never think of stealing again in fact I feel ashamed and I wonder how I was able to do it before. Now I don't have money for my meals and my transport to here. This is indeed a problem and can make us go back to square one again (male 37 years, married, ordinary diploma graduate, unemployed on methadone for 19months).

\section{Discussion}

The study found that it is quite extraordinary that with only two years of implementation of MAT in Muhimbili National Hospital, Dares-Salaam region-Tanzania, the program is faced with almost similar challenges with developed countries which have had methadone in place for quite some time now. Nevertheless, some differences exist on the characteristics of the challenges report by the study. The study has highlighted challenges of accessing and maintaining treatment by the study participants.

Strict admission criterion is perhaps the strongest challenge in accessing treatment found by this study. Muhimbili clinic receives clients who only injects while some countries receive clients regardless of their route of administration. ${ }^{17}$ The study participants reported shifting their route of administration from oral route to injection route just to be accepted into the program. This can be explained by strict program policy which has got huge implication on the HIV epidemic by accelerating it rather than combating it. Therefore, urgent review of the program policy by the program stakeholders is called for to rescue the population of opioid users who are falling into injection for the sake of being accepted into MAT programs. Therefore the admission criteria should be less strict to accept clients regardless of their route of drug use.

Logistic difficulties related to unemployment are another important challenge in accessing MAT services. Methadone at the Muhimbili clinic is provided free of charge to lessen the burden of cost for consumers, but still the burden of costs is huge because the clinic requires the consumers to attend on the daily basis which pose difficulties in affording bus fair as most of them are unemployed. While in the United States and Western European countries allow take home methadone doses for stable patients in order to solve the logistic difficulties, this is prohibited in most countries of Eastern Europe, Central Asia and Tanzania. Although there are therapeutic benefits of daily attendance to MAT clinics, take home doses has been associated with increased retention in treatment. ${ }^{18}$
Studies report that rapid access to treatment is associated with retention in treatment. ${ }^{19}$ This study however found that patients can stay up to three hours waiting for treatment and also the clinic has a fixed time of operation (6am to mid day) which threats the future retention of the patients in the program and even deter others from joining the program. Waiting for longer period at the clinic has also been reported in other study in the USA, China, Russia and Canada ${ }^{20,21}$ in which the factor contributed to patients quitting the program. Waiting for long time could be explained by shortage of workers and lack of enough space to dispense methadone at the clinic, instead only one window is open to cater for more than 400 clients who come for treatment on a daily basis without missing. To deliver treatment effectively medication assisted treatment programs need adequate human resources and low staff turnover ${ }^{22}$ which is not the case at the Muhimbili MAT clinic. The clinic should adopt client/patient centered program policies to ensure that there are flexible hours of operation (to extend the hours of operation) and also to ensure that clients receive treatment as rapidly as possible and the waiting period to be as short as possible within the realm of good practice.

Study participants also reported to be unable to move between the two clinics found in Dar es Salaam. This is however different in other countries like china where referral between the local clinics is available though poor. Lack of transferability has been reported in other studies to cause poor treatment retention. ${ }^{11}$ The program stakeholders should ensure local program transferability. Clients should be able to move within their community (Muhimbili, Mwananyamala and Temeke) and still have access to treatment

Moreover, drug use is culturally unacceptable for many societies in Tanzania. The study found some stigma and some misconceptions about methadone. Ongoing stigma and the misconception can discourage the service users from joining or maintaining treatment in the future. The stigma and the misconception could be due to lack of education about drug use and MAT programs by the society. This finding was also reported in some studies in china ${ }^{5}$ and in the USA by. ${ }^{23}$ The program stakeholders should ensuring strong community awareness campaigns to address the issue of stigma and methadone myths.

Adjustment to new life is reported in this study to be another important challenge the clients encounter. This is could be due to lack of multisectoral collaboration in implementation of MAT program as recommended by other studies. ${ }^{22}$ The researcher perceives that this can lead the service users into the previous behaviors of theft in order to get the living or afford to get to the clinic. On the other hand the services users are likely to quit use of MAT services and go back to the previous life style making the viscous cycle if at all urgent interventions are not done to help the service users cope with life hardships. The program stakeholders should ensure that the Muhimbili MAT is directly linked to a variety of other services and supports that a client/patient may need like educational programs, employment programs (vocational skills/training, employment services) and spiritual organizations. These linkages can be a critical aspect of ensuring continuity of care for clients and also it can prevent them from slipping through the cracks.

Furthermore, the public security authorities in Tanzania still regard drug abuse from a criminal justice point of view which complicates the utilization of MAT for the users. The costs of receiving methadone have often included harassment from law enforcement. In this study, 
the service users reported police harassment to be a big disincentive to treatment. Tanzania is not the only country faced by this problem. Similar problem has been reported in Kyrgyzstan and Ukraine that police often arrested patients or threaten to plant drugs on them unless they paid bribes. ${ }^{24}$ This constant harassment from police could be due to poor involvement of the police in MAT programs and poor attitudes of the police towards opiate users which might have a bad implication to the program future. Therefore the program stakeholders should ensure active involvement of police in the MAT program. ${ }^{25}$

\section{Limitations and strength of the study}

This was a qualitative study therefore the findings cannot be generalized to all opiate users receiving MAT services in Dar-esSalaam region, Tanzania. Also the recruitment procedure restricted the researcher from finding hard to reach opiate users who have never used MAT services, who could have more insight of the challenges of accessing this service. Even with these limitations, the study has highlighted the specific information on the characteristics of challenges in accessing MAT services in Tanzania that can inform improvements in this program.

Also the highlighted experiences from this study can help to inform local scale up as well as initiatives in other African settings with generalized HIV and heroin abuse epidemics in designing and providing comprehensive and evidence-based services for persons with injecting drug use (PWID), where injection drug use is identified as contributing to the spread of the HIV epidemic.

\section{Conclusion}

The study has revealed the challenges against accessing MAT services in Muhimbili National Hospital, Dar-es-Salaam regionTanzania. It is therefore essential for different stakeholders to recognize and respond to these barriers because as the MAT programs improve, thousands of opiate users in Tanzania and the Tanzanians as a whole will benefit.

\section{Recommendation}

This MAT programme is a good one as it has shown some significant general health as well as mental health improvement among drug injectors and drug abusers in Muhimbili National Hospital, Dar-esSalaam region-Tanzania. Since it is a pilot the Tanzania government through the Ministry of Health \& Social Welfare in collaboration with the Drug Control Commission in the Prime Minister's Office and interested International partners such as CDC, USAID, NIDA etc should increase efforts of funding so that this important and necessary service can be extended to other growing regions like the Mirembe National Mental Hospital in Dodoma region which is the growing political capital of the Tanzania and drug abuse is an increasing public health problem in this region among adolescents and even quick statistics of hospital admissions in Mirembe National Hospital is mostly due to a wide range of substances abuse.

Furthermore, if Methadone treatment is expensive, other developed countries have tried as well another similar but less potent compared to methadone but produces some relief to drug abusers and drug injectors. This drug is called buprenorphine was commonly used in the US among drug abusers but was reported to be not quite potent and thus not widely used. ${ }^{25}$ So it may be wealth considering introducing this drug as well to be piloted in various regions in Tanzania as in the
US drug abusers majority use heroin and cocaine, whereas in Tanzania the majority of the victims use marijuana (Bhangi) and to relatively small extent cocaine, heroin and mandrax etc or combine these drugs in order for the users getting high quickly.

\section{Acknowledgements}

Role of funding source: the author declares that no financial assistance was received. The author would like to thank the management and staff of Muhimbili methadone clinic for their help and support. The study would not have been possible without the study participants. We thank them very much.

Contributors; Olimpia Laswai conceived the study idea, collected the data, conducted the analysis and wrote the report. Dr Stephen ED Nsimba $(\mathrm{PhD})$, assisted in the analysis of the data and reviewing the report and rewriting a manuscript.

\section{Conflict of interest}

The authors declare that there is no conflict of interest.

\section{References}

1. Helen J Levine, Sharon Reif, Margaret T Lee, et al. Alcohol and Drug Services Study. The National Treatment System: Outpatient Methadone Facilities. Office of applied studies, Rockville, 2004. p. 1-64.

2. Dole VP, Nyswander ME. Methadone Maintenance Treatment: A ten syears perspective. JAMA. 1976;235(19):2117-2119.

3. Michels II, Stöver H, Gerlach R. Substitution treatment for opioid addicts in Germany. Harm Reduct J. 2007;4:5 p.

4. Schreuder I, van der Sande MA, de Wit M, et al. Seroprevalence of HIV, hepatitis B, and hepatitis $\mathrm{C}$ among opioid drug users on methadone treatment in the Netherlands. Harm Reduct J. 2010;7:25.

5. Lin $\mathrm{C}, \mathrm{Wu} \mathrm{Z}$, Rou $\mathrm{K}$, et al. Challenges in providing service in methadone maintenance therapy clinics in China: Service providers' perceptions. Int J Drug Policy. 2010;21(3):173-178.

6. Mohamad N, Bakar NH, Musa N, et al. Better retention of Malaysian opiate dependents treated with high dose methadone in methadone maintenance therapy. Harm Reduct J. 2010;7:30.

7. Morineau G, Bollen LJ, Syafitri RI, et al. HIV prevalence and risk behaviours among injecting drug users in six Indonesian cities implications for future HIV prevention programs. Harm Reduct J. 2012;9:37

8. Jardine M, Thi Nguyen VA, Khuat TH. Case study: Methadone maintenance treatment in Hanoi, Vietnam. Harm Reduct J. 2012;9:26.

9. Williams ML, McCurdy SA, Bowen AM, et al. HIV Seroprevalence in a Sample of Tanzanian Intravenous Drug Users. AIDS Educ Prev. 2009;21(5):474-483.

10. Donald G McNeil. Addiction: A first in Sub Saharan Africa: Methadone Maintenance Program. New York Times, Washington, USA, 2011.

11. Wu F, Peng CY, Jiang H, et al. Methadone maintenance treatment in China: perceived challenges from the perspectives of service providers and patients. J Public Health (Oxf). 2013;35(2):206-212.

12. Wood L, Haber J. Nursing research: Methods, critical appraisal and utilization. 5th ed. St. Louis, USA: Mosby; 2002.

13. Tanzania Demographic Profile. Tanzania, 2016.

14. Masao F, Nyandindi C, Buma D, et al. Standard Operating Procedures (SOPs) for Medically Assisted Therapy (MAT). 2012. (Unpublished) 
15. White Marilyn Domas, Marsh Emily E. Content analysis: A flexible methodology. Library trends. 2006;55(1):22-45.

16. Graneheim UH, Lundman B. Qualitative content analysis in nursing research: Concepts, procedures and measures to achieve trustworthiness. Nurse Educ Today. 2004;24(2):105-112.

17. Public Health Fact Sheet. USA: APHA; 2013.

18. Lowinson JH, Payte JT, Salsitz E, et al. Methadone maintenance. 3rd ed Baltimore, Maryland, USA: Williams and Wilkins; 1997. p. 405-415.

19. Ward Jeff, Richard P Mattick, Hall Wayne. How long is long enough? Answers to questions about the duration of methadone maintenance treatment. In: Jeff Ward, Richard P Mattick, et al. editors. Methadone maintenance treatment and other opioid replacement therapies. Netherlands: Harwood Academic Press; 1998. p. 305-336.

20. Hobden KL, Cunningham JA. Barriers to the dissemination of four harm reduction strategies: A survey of addiction treatment providers in Ontario. Harm Reduct J. 2006;3:35 p.
21. Rettig RA, Yarmolinsky A. Federal regulation of methadone treatment. USA: National Academy Press; 1995.

22. Jamieson, Beals, Lalonde. Best Practices: Methadone Maintenance Treatment. Ontario, Canada: Health Canada Ottawa; 2002.

23. Zaller ND, Bazazi AR, Velazquez L, et al. Attitudes toward Methadone among Out-of-Treatment Minority Injection Drug Users. Int J Environ Res Public Health. 2009;6(2):787-797.

24. Spicer N, Bogdan D, Brugha R, et al. 'It's risky to walk in the city with syringes': understanding access to HIV/AIDS services for injecting drug users in the former Soviet Union countries of Ukraine and Kyrgyzstan. Global Health. 2011;7:22.

25. Nsimba SED. Methadone Maintenance Therapy: A review paper of the summary of evidence based approach for long patient waiting time for this service in the United States. East African Journal of Public Health. 2010;7:54-57. 